\title{
Long non-coding RNA LUCAT1 contributes to cisplatin resistance by regulating the miR-514a-3p/ULK1 axis in human non-small cell lung cancer
}

\author{
QIMING SHEN, ZHE XU and SHUN XU
}

Department of Thoracic Surgery, The First Hospital of China Medical University, Shenyang, Liaoning 110001, P.R. China

Received October 11, 2019; Accepted July 15, 2020

DOI: $10.3892 /$ ijo.2020.5106

\begin{abstract}
Drug resistance is a major obstacle in the therapy of malignant tumors, including non-small cell lung cancer (NSCLC). Long non-coding RNAs (lncRNAs) have been demonstrated to be involved in chemoresistance. The present study aimed to investigate the role of lung cancer-associated transcript 1 (LUCAT1) in cisplatin (DDP) resistance in NSCLC. By using reverse transcription-quantitative polymerase chain reaction (RT-qPCR), it was found that the expression of LUCAT1 was elevated and that of microRNA-514a-3p (miR-514a-3p) was decreased in DDP-resistant NSCLC tissues and cells. Functionally, LUCAT1 upregulation enhanced cisplatin resistance by promoting the viability, autophagy and metastasis, and inhibiting the apoptosis of NSCLC cells, as demonstrated by Cell Counting kit-8 (CCK-8) assay, western blot analysis, Transwell assay and flow cytometric analysis. LUCAT1 was identified as a sponge of miR-514a-3p and uncoordinated-51-like kinase 1 (ULK1) was proven to be a target gene of miR-514a-3p by bioinformatics analysis, dual-luciferase reporter assay and RNA immunoprecipitation (RIP) assay. The enhancing effect of miR-514a-3p on cisplatin sensitivity was reversed by the elevation of LUCAT1. ULK1 knockdown suppressed cisplatin resistance, while this effect was attenuated by miR-514a-3p inhibition. Moreover, LUCAT1 positively regulated ULK1 expression by targeting miR-514a-3p. In addition, LUCAT1 knockdown suppressed tumor growth in vivo. On the whole, the findings of the present study demonstrate that LUCAT1 contributes to the resistance of NSCLC cells to cisplatin by regulating the miR-514a-3p/ULK1 axis, elucidating a novel regulatory network in cisplatin resistance in NSCLC.
\end{abstract}

Correspondence to: Dr Qiming Shen, Department of Thoracic Surgery, The First Hospital of China Medical University, 155 North Nanjing Road, Heping, Shenyang, Liaoning 110001, P.R. China E-mail: qendjj@163.com

Key words: non-small cell lung cancer, LUCAT1, miR-514a-3p, ULK1, cisplatin resistance

\section{Introduction}

Non-small cell lung cancer (NSCLC) is a type of lung cancer that severely threatens the health and life of individuals worldwide $(1,2)$. Although researchers have made significant progress in early diagnostic and treatment methods in recent years, the prognosis of patients with NSCLC remains unsatisfactory $(3,4)$. Chemotherapy is one of the main approaches used in the treatment of NSCLC, and cisplatin (DDP) is a common drug used in the treatment of NSCLC $(5,6)$. However, the acquisition of chemoresistance severely hinders the effects of chemotherapy (7). Therefore, it is particularly essential to explore the mechanisms responsible for drug resistance in NSCLC.

Long non-coding RNAs (lncRNAs) are a set of non-coding RNAs (ncRNAs) of $>200$ nucleotides (nts) in length, which do not have protein-coding potential (8). Recently, an increasing number of lncRNAs have been elaborated, and studies have demonstrated that diverse lncRNAs play crucial roles in drug resistance in NSCLC. For instance, Ge et al indicated that FOXD2 adjacent opposite strand RNA 1 (FOXD2-AS1) was aberrantly expressed in drug-resistant NSCLC and that its absence suppressed cisplatin resistance in cisplatin-resistant NSCLC cells (9). Liu et al demonstrated that HOX transcript antisense RNA (HOTAIR) was upregulated in cisplatinresistant NSCLC patients and the deficiency of HOTAIR improved cisplatin sensitivity in cisplatin-resistant NSCLC cells (10). However, Wang et al found that the maternally expressed 3 (MEG3) level was decreased in patients with cisplatin-resistant NSCLC and that the elevation of MEG3 enhanced the sensitivity of NSCLC cells to cisplatin (11). These studies suggest that lncRNAs play dual roles in regulating drug resistance in NSCLC. The present study focused on the function of lung cancer-associated transcript 1 (LUCAT1) in cisplatin resistance in NSCLC.

MicroRNAs (miRNAs or miRs), a family of ncRNAs of approximately $22 \mathrm{nts}$ in length, which modulate gene expression by recognizing the 3 '-untranslated region (3'UTR) of target messenger RNAs (mRNAs) (12). An increasing number of miRNAs have been confirmed to function as vital mediators of drug resistance in human tumors, including NSCLC. For example, miR-197 has been shown to be weakly expressed in patients with platinum-resistant NSCLC and miR-197 inhibition has been shown to enhance drug resistance and tumor 
growth (13). It has also been demonstrated that the upregulation of miR-451 suppresses the resistance of A549 cells to DDP by inhibiting cell growth and inducing cell apoptosis (14). The deficiency of miR-138-5p also contributes to the resistance of NSCLC cells to gefitinib (15). Nevertheless, to the best of our knowledge, there are no studies available to date on the role of miR-514a-3p in DDP resistance in NSCLC.

Uncoordinated-51-like kinase 1 (ULK1) is an autophagy-related gene which has been revealed to play a role in the progression of drug resistance in diverse human cancers, such as hepatocellular carcinoma (HCC) (16), breast cancer (17) and colorectal cancer (18). Moreover, Zhao et al proved that claudin 1 (CLDN1) enhanced drug resistance via the phosphorylation of ULK1 in NSCLC (19), indicating that ULK1 plays a vital role in drug resistance in NSCLC.

In the present study, the expression levels of LUCAT1, miR-514a-3p and ULK1 in cisplatin-resistant NSCLC cells were investigated. Furthermore, the functions and underlying mechanisms of LUCAT1 in the resistance of NSCLC cells to DDP were explored.

\section{Materials and methods}

Tissue collection. After the patients received DDP treatment, a total of 30 DDP-resistant NSCLC tissues, 30 DDP-sensitive NSCLC tissues and 30 tumor-adjacent normal tissues were harvested from patients with NSCLC who were resistant or sensitive to DDP at the First Hospital of China Medical University between October, 2015 and June, 2017. All patients with NSCLC received DDP-based treatment for 6 cycles. The clinicopathological characteristics of the patients with NSCLC are presented in Table I. The samples were immediately placed in liquid nitrogen and preserved at $-80^{\circ} \mathrm{C}$ until use. The sample collection was conducted under the supervision of the Ethics Committee of the First Hospital of China Medical University. Written informed consent forms were signed by the patients.

Cells and cell culture. Normal human lung fibroblasts (IMR90) and NSCLC cells (A549) were purchased from the American Type Culture Collection (ATCC). To establish DDP-resistant NSCLC cells (A549/DDP), A549 cells were exposed to gradually increasing concentrations of cisplatin (Beijing Solarbio Science \& Technology Co., Ltd.) until the cells were able to proliferate stably. All the cells were cultured in RPMI-1640 medium (Gibco; Thermo Fisher Scientific, Inc.) supplemented with $10 \%$ fetal bovine serum (FBS; Gibco; Thermo Fisher Scientific, Inc.) and $1 \%$ penicillin/streptomycin (Beijing Solarbio Science \& Technology Co., Ltd.) in an incubator containing $5 \% \mathrm{CO}_{2}$ at $37^{\circ} \mathrm{C}$.

Cell transfection. The overexpression vector of LUCAT1 (LUCAT1), the overexpression vector of ULK1 (ULK1) and their control (Vector), short hairpin RNA (shRNA) targeting LUCAT1 (sh-LUCAT1), shRNA targeting ULK1 (sh-ULK1) and their control (sh-NC), mimics of miR-514a-3p (miR-514a-3p) and its control (miR-NC), inhibitors of miR-514a-3p (Anti-miR-514a-3p) and its control (Anti-NC) were synthesized by Guangzhou RiboBio Co., Ltd. 50 nM synthetic oligonucleotides or $2 \mu \mathrm{g}$ vectors were transfected into A549 or A549/DDP cells utilizing Lipofectamine 2000 (Invitrogen; Thermo Fisher Scientific, Inc.) according to the manufacturers' instructions. Following 48 h of transfection, the cells were harvested for further experiments. RT-qPCR or western blot analysis were conducted to determine the transfection efficiency.

A549 cells were transfected with LUCAT1, Vector, Anti-NC, Anti-miR-514a-3p, Anti-miR-514a-3p + sh-NC, Anti-miR-514a-3p + sh-LUCAT1, ULK1, ULK1 + miR-NC, ULK1 + miR-514a-3p, LUCAT1+miR-NC or LUCAT1 + miR514a-3p and then treated with or without cisplatin. A549/DDP cells were transfected with sh-LUCAT1, sh-NC, miR-NC, miR-514a-3p, miR-514a-3p + Vector, miR-514a-3p + LUCAT1, sh-ULK1, sh-ULK1 + Anti-NC or sh-ULK1 + Anti-miR-514a-3p and then treated with or without cisplatin.

Reverse transcription-quantitative polymerase chain reaction $(R T-q P C R)$. Total RNA was extracted from the tissues and cells using RNAiso Plus (Takara Biotechnology Co., Ltd.). The abundance of RNA samples was measured using a NanoDrop 2000c spectrophotometer (Thermo Fisher Scientific, Inc.). Reverse transcription was then conducted using the PrimeScript $^{\mathrm{TM}}$ RT reagent kit (Takara Biotechnology Co., Ltd.) or the miRNA 1st Strand cDNA Synthesis kit (Vazyme Biotech Co., Ltd.). Subsequently, qPCR was performed with AceQ Universal SYBR qPCR Master Mix (Vazyme Biotech Co., Ltd.) under the following thermocycling conditions: i) $95^{\circ} \mathrm{C}$ for $5 \mathrm{~min}$; ii) 40 cycles of $95^{\circ} \mathrm{C}$ for $10 \mathrm{sec}$ and $60^{\circ} \mathrm{C}$ for $30 \mathrm{sec}$; iii) $95^{\circ} \mathrm{C}$ for $15 \mathrm{sec}, 60^{\circ} \mathrm{C}$ for $60 \mathrm{sec}$ and $95^{\circ} \mathrm{C}$ for $15 \mathrm{sec}$. The levels of LUCAT1, miR-514a-3p and ULK1 were evaluated using the $2^{-\Delta \Delta \mathrm{Cq}}$ method (20). Glyceraldehyde 3-phosphate dehydrogenase (GAPDH) was used to normalize the expression of LUCAT1 and ULK1, while small nuclear RNA U6 was used to normalize the expression of miR-514a-3p. The primers used were as follows: LUCAT1 forward, 5'-ACCAGC TGTCCCTCAGTGTTCT-3' and reverse, 5'-AGGCCTTTA TCCTCGGGTTGCCT-3'; miR-514a-3p forward, 5'-ATTGAC ACTTCTGTGAGTAGA-3' and reverse, 5'-CAGTGCGTG TCGTGGAGT-3'); ULK1 forward, 5'-TGCCCCTGGTTG AATGTTCT-3' and reverse, 5'-ACACCAGCCCAACAATTC CA-3'; GAPDH forward, 5'-GGTCTCCTCTGACTTCAA CA-3' and reverse, 5'-GTGAGGGTCTCTCTCTTCCT-3'); and U6 forward, 5'-TGCGGGTGCTCGCTTCGGCAGC-3' and reverse, 5'-CCAGTGCAGGGTCCGAGGT-3'.

Cell Counting kit-8 (CCK-8) assay. For the analysis of cisplatin resistance, A549 and A549/DDP cells were seeded into 96-well plates and exposed to various concentrations of cisplatin $(0.5,1.5$ or $2.0 \mu \mathrm{g} / \mathrm{ml}$; Beijing Solarbio Science \& Technology Co., Ltd.) for 24 h. Subsequently, $20 \mu$ l CCK-8 solution (Beyotime Institute of Biotechnology, Inc.) was added to each well and cultured for a further $2 \mathrm{~h}$ at $37^{\circ} \mathrm{C}$. The absorbance was examined at $450 \mathrm{~nm}$ using a microplate reader (Bio-Rad Laboratories, Inc.). The half maximal inhibitory concentration $\left(\mathrm{IC}_{50}\right)$ was the concentration of cisplatin that induced $50 \%$ growth inhibition and determined by the relative survival curve. For the analysis of cell viability, CCK-8 (Beyotime Institute of Biotechnology, Inc.) was added at $0,24,48$ and $72 \mathrm{~h}$. The other steps were the same as those described above. 
Table I. Clinicopathological characteristics of the patients with NSCLC.

\begin{tabular}{|c|c|c|c|c|}
\hline \multirow[b]{2}{*}{ Parameter } & \multirow[b]{2}{*}{ Case } & \multicolumn{2}{|c|}{ NSCLC patients } & \multirow[b]{2}{*}{ P-value } \\
\hline & & DDP-resistant $(\mathrm{n}=30)$ & DDP-sensitive $(\mathrm{n}=30)$ & \\
\hline \multicolumn{5}{|l|}{ Age (years) } \\
\hline$\leq 65$ & 32 & 17 & 15 & 0.6048 \\
\hline$>65$ & 28 & 13 & 15 & \\
\hline \multicolumn{5}{|l|}{ Sex } \\
\hline Male & 32 & 14 & 18 & 0.3006 \\
\hline Female & 28 & 16 & 12 & \\
\hline \multicolumn{5}{|c|}{ Lymph node metastasis } \\
\hline No & 22 & 7 & 15 & $0.0321^{\mathrm{a}}$ \\
\hline Yes & 38 & 23 & 15 & \\
\hline \multicolumn{5}{|l|}{ Stage } \\
\hline $\mathrm{I}+\mathrm{II}$ & 35 & 13 & 22 & $0.0184^{\mathrm{a}}$ \\
\hline III & 25 & 17 & 8 & \\
\hline
\end{tabular}

${ }^{\text {ap }}<0.05$ vs. no lymph node metastasis or Stage I+II.

Flow cytometric analysis. The Annexin V-fluorescein isothiocyanate (FITC)/propidium iodide (PI) Apoptosis Detection kit (Beyotime Institute of Biotechnology, Inc.) was utilized for the analysis of cell apoptosis. In brief, cells were harvested, washed, resuspended, and then $5 \mu \mathrm{l}$ AnnexinV-FITC and $10 \mu \mathrm{l}$ PI were added and maintained for $15 \mathrm{~min}$ in the dark to stain the cells. The stained cells were analyzed using a flow cytometry (BD Biosciences).

Western blot analysis. Total protein was isolated from the cells using RIPA buffer (Beyotime Institute of Biotechnology, Inc.). The proteins were quantified using a NanoDrop $2000 \mathrm{c}$ spectrophotometer (Thermo Fisher Scientific, Inc.). A total of $20 \mu \mathrm{g}$ proteins were separated by $10 \%$ sodium dodecyl sulfonate-polyacrylamide gel (SDS-PAGE; Beijing Solarbio Science \& Technology Co., Ltd.) and then transferred onto polyvinylidene difluoride membranes (PVDF; Pall Corporation). The membranes were blocked in skim milk for $1 \mathrm{~h}$ at room temperature and incubated with primary antibodies against microtubule-associated protein light chain 3-I/II (LC3-I/II; ab192890; 1:2,000; Abcam), p62 (ab56416; 1:2,000; Abcam), ULK1 (ab167139; 1:1,000; Abcam) or GAPDH (ab9485; 1:2,500; Abcam) overnight at $4^{\circ} \mathrm{C}$ followed by incubation with corresponding secondary antibody (ab150117; 1:2,000; Abcam) for $2 \mathrm{~h}$ at room temperature. The protein levels were analyzed using an enhanced chemiluminescence kit (Vazyme Biotech Co., Ltd.). The results were analyzed using software ImageJ v1.8.0 (National Institutes of Health).

Transwell assay. Transwell chambers (Corning, Inc.) coated with (for cell invasion assay) or without (for cell migration assay) Matrigel (Beijing Solarbio Science \& Technology Co., Ltd.) were employed to examine cell invasion and migration. Briefly, following relevant transfection and treatment, the A549 or A549/DDP cells in serum-free medium were added to the upper chamber and culture medium supplemented with
10\% FBS (Gibco; Thermo Fisher Scientific, Inc.) was added to the bottom chamber. After $24 \mathrm{~h}$, the migrated or invaded cells were fixed with methanol and stained with $0.1 \%$ crystal violet (Beijing Solarbio Science \& Technology Co., Ltd.) for $20 \mathrm{~min}$ at room temperature. The stained cells were observed under an inverted microscope (Olympus Corporation).

Dual-luciferase reporter assay. The potential binding sites between miR-514a-3p and LUCAT1 or ULK1 were predicated by StarBase 3.0 (http://starbase.sysu.edu.cn/tutorialAPI. php) and then verified by dual-luciferase reporter assay. In brief, the sequences of LUCAT1 or 3'UTR of ULK1 containing the potential binding sites of miR-514a-3p were amplified and introduced into the XhoI and XbaI sites of the downstream of Firefly luciferase gene in the pmirGLO vector (Promega Corporation) to generate luciferase reporter vectors LUCAT1-WT and ULK1-3'UTR-WT, respectively. The sequences of mutant type LUCAT1 or 3'UTR of ULK1 lacking the binding sites of miR-514a-3p were also cloned into the pmirGLO vector to construct the reporter vectors, LUCAT1-MUT and ULK1-3'UTR-MUT, respectively. The A549 cells and A549/DDP cells were seeded into 24-well plates and $100 \mathrm{ng}$ of the indicated luciferase reporter vector were then transfected into the cells in combination with $50 \mathrm{nM}$ miR-514a-3p or miR-NC using Lipofectamine 2000 (Invitrogen; Thermo Fisher Scientific, Inc.). After 48 h, the cells were collected and the luciferase activity was determined using a Dual-Luciferase Reporter Assay kit (Promega Corporation). Renilla luciferase activity was used to normalize Firefly luciferase activity.

RNA immunoprecipitation (RIP) assay. RIP assay was conducted using a Magna RIP ${ }^{\text {TM }}$ RNA Binding Protein Immunoprecipitation kit (EMD Millipore). In brief, the A549 cells or A549/DDP cells were lysed with RIP lysis buffer and then incubated overnight at $4^{\circ} \mathrm{C}$ with magnetic beads 
A
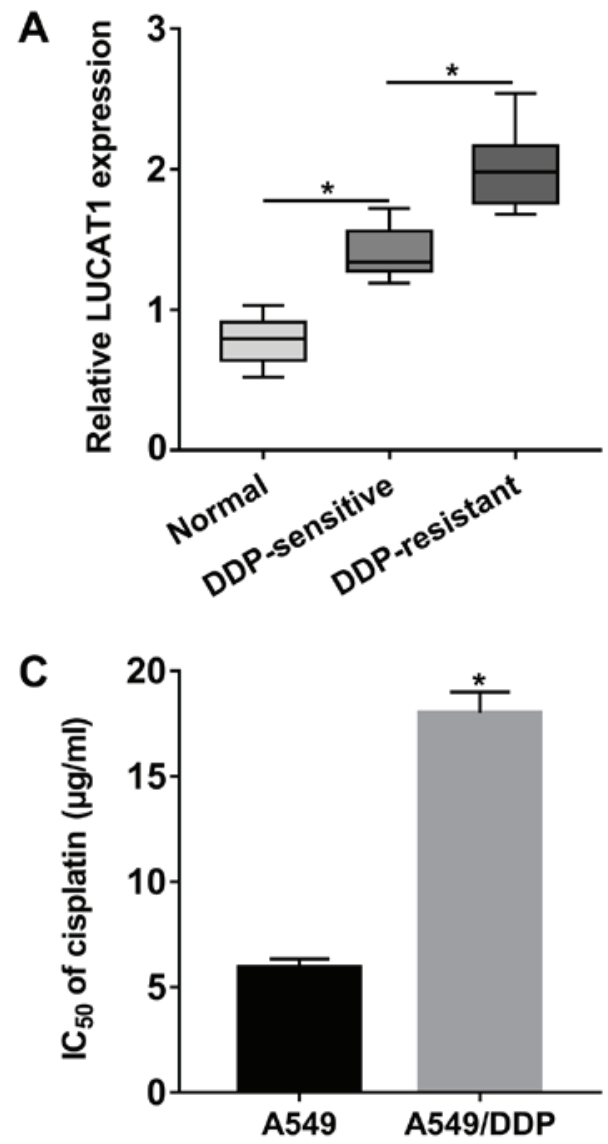

B
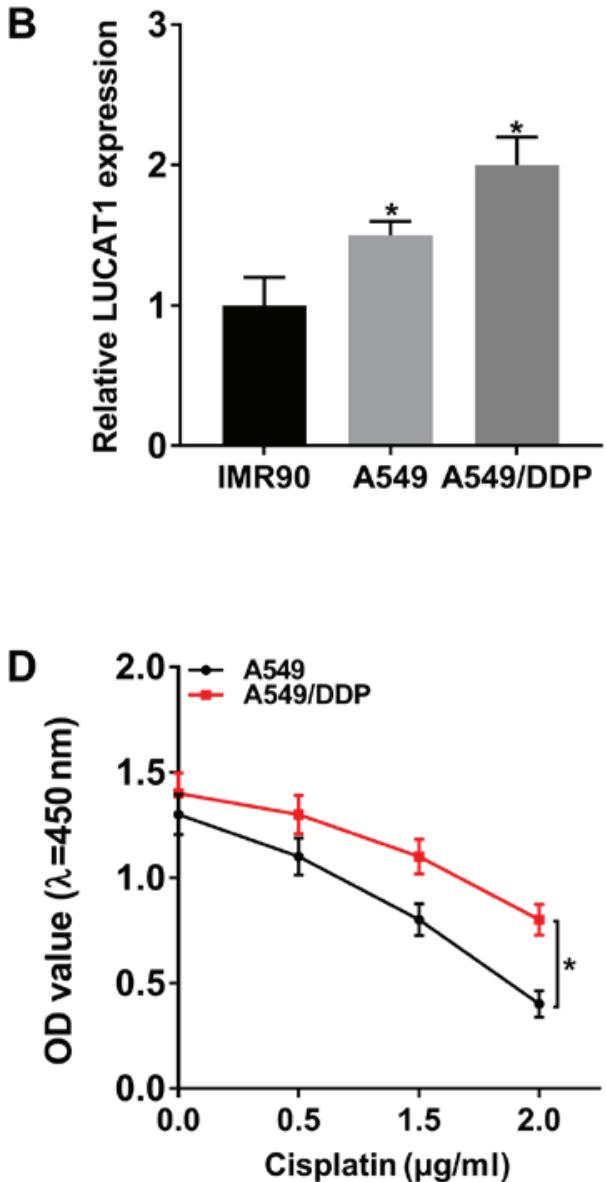

Figure 1. High expression of LUCAT1 in DDP-resistant NSCLC tissues and cells. (A) The expression of LUCAT1 in normal tissues, DDP-sensitive NSCLC tissues and DDP-resistant NSCLC tissues was examined by RT-qPCR. (B) The expression of LUCAT1 in IMR90, A549 and A549/DDP cells was measured by RT-qPCR. (C) IC $_{50}$ value of cisplatin in A549 and A549/DDP cells was determined by CCK-8 assay. (D) The viability of the A549 and A549/DDP cells was detected by CCK-8 assay. "P<0.05 vs. respective control. LUCAT1, lung cancer-associated transcript 1; DDP, cisplatin; NSCLC, non-small cell lung cancer.

conjugated with antibody against Argonaute2 (Anti-Ago2; ab32381; 1:2,000; Abcam) or immunoglobulin G (Anti-IgG; ab109489; 1:5,000; Abcam). The cells were incubated with Proteinase K (Beijing Solarbio Science \& Technology Co., Ltd.) for $30 \mathrm{~min}$ at $55^{\circ} \mathrm{C}$. Finally, the enrichment of LUCAT1, miR-514a-3p and ULK1 was measured by RT-qPCR following the purification of the RNA as described above.

In vivo experiment. A total of 28 male nude mice (age: 5 weeks old; weight: 16-23 g) were obtained from Shanghai SLAC Laboratory Animals Co., Ltd. and divided into 4 groups $(\mathrm{n}=7 /$ group $)$ : Vector + Cisplatin, LUCAT1 + Cisplatin, sh-NC + Cisplatin and sh-LUCAT1 + Cisplatin. All the mice were housed at $27^{\circ} \mathrm{C}$ in pathogen-free conditions with $45 \%$ humidity and $12 \mathrm{~h}$ light/dark cycle and fed sterile fodder and drinking water. For LUCAT1 + Cisplatin and Vector + Cisplatin groups, the A549 cells were transfected with LUCAT1 or Vector and then injected subcutaneously into the flanks of the mice. For sh-LUCAT1 + Cisplatin and sh-NC + Cisplatin groups, the A549/DDP cells were transfected with sh-LUCAT1 or sh-NC, and then injected subcutaneously into the flanks of the mice. After 1 week, all mice were administered cisplatin $(5 \mathrm{mg} / \mathrm{kg}$; Beijing Solarbio Science \& Technology Co., Ltd.) each week for 5 weeks. Tumor volume was detected each week and calculated using the formula: (length $\mathrm{x}$ width $\left.{ }^{2}\right) / 2$. After 6 weeks, the mice were euthanized by cervical dislocation and tumors were harvested, weighed and preserved at $-80^{\circ} \mathrm{C}$ for analysis by RT-qPCR. The criteria for judging the death of the mice were continuous involuntary breathing for 2-3 min and no blinking reflex. The animal experiments were approved by the Ethics Committee of Animal Research of the First Hospital of China Medical University. The humane endpoints established in the present study for the animal experiments were as follows: A tumor burden $>10 \%$ of the body weight and a tumor which did not exceed $20 \mathrm{~mm}$ in any one dimension.

Statistical analysis. All data were obtained from 3 independent experiments and are presented as the means \pm standard deviation (SD). GraphPad Prism 7 software (GraphPad Inc.) was employed to analyze the collected data. A paired/unpaired Student's t-test or one-way analysis of variance (ANOVA) followed by Tukey's test were utilized to determine significant differences between groups. The clinicopathological features were analyzed using an $\chi^{2}$ test. A value of $<0.05$ was considered to indicate a statistically significant difference.

\section{Results}

LUCAT1 is highly expressed in DDP-resistant NSCLC tissues and cells. In order to investigate the potential role 
A

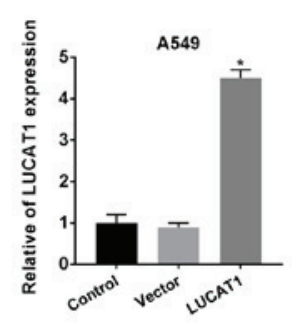

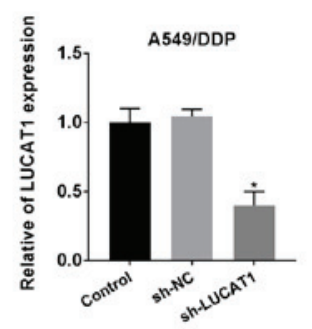

B
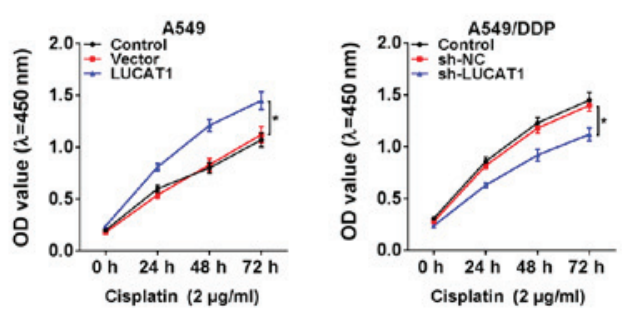

C
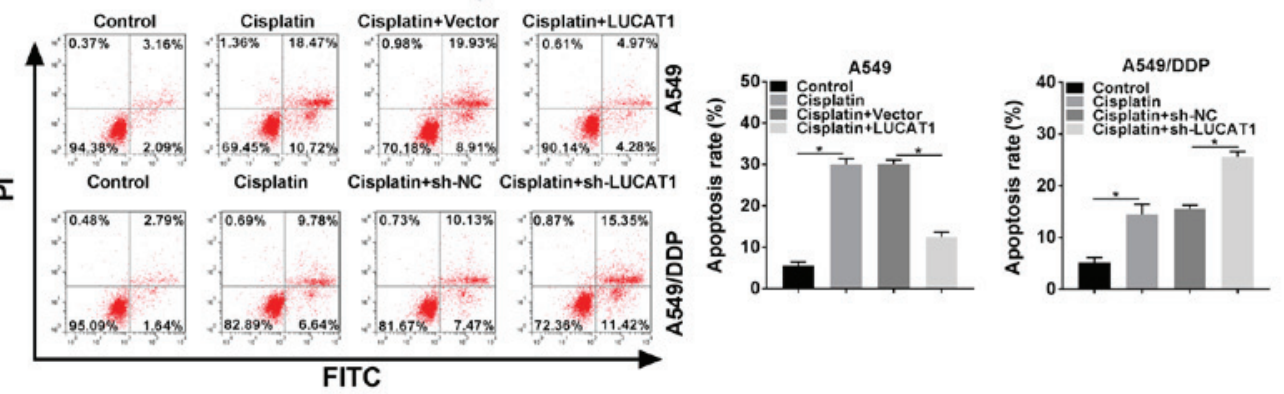

D
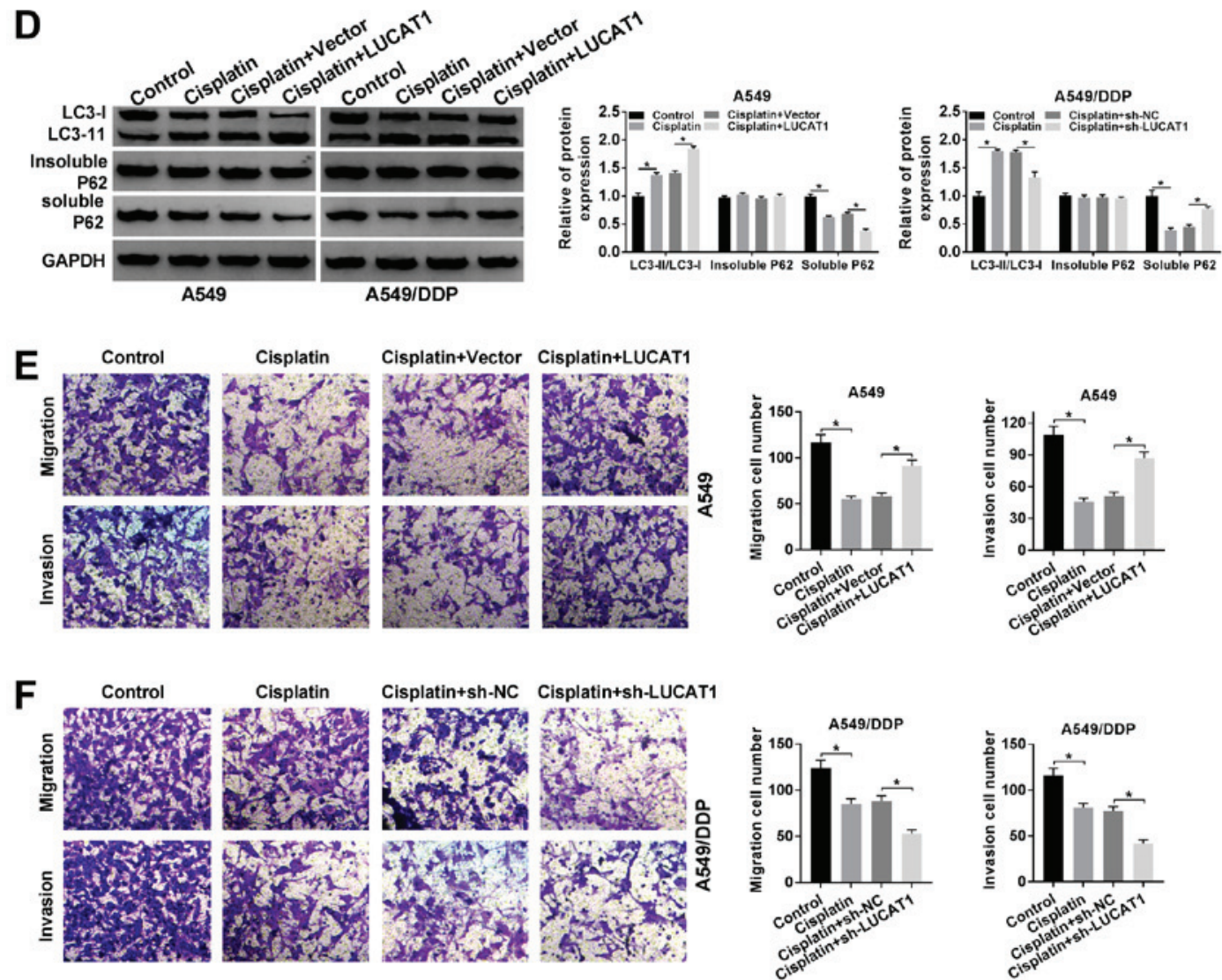

Figure 2. LUCAT1 plays a role in the regulation of cisplatin resistance by modulating the apoptosis, autophagy, migration and invasion of NSCLC cells. (A and B) A549 cells were transfected with Vector or LUCAT1, A549/DDP cells were transfected with sh-NC or sh-LUCAT1, and untransfected A549 cells and A549/DDP cells were used as the control groups. (A) The level of LUCAT1 in A549 cells and A549/DDP cells was measured by RT-qPCR. (B) The viability of A549 cells and A549/DDP cells was assessed through CCK-8 assay. (C-F) A549 cells were treated with cisplatin, cisplatin + Vector or cisplatin + LUCAT1, A549/DDP cells were treated with cisplatin, cisplatin + sh-NC or cisplatin + sh-LUCAT1, and untreated A549 cells and A549/DDP cells were used as the control group. (C) The apoptosis of A549 cells and A549/DDP cells was evaluated by flow cytometric analysis. (D) The ratio of LC3-II/LC3-I and the levels of insoluble p62 and soluble p62 in A549 cells and A549/DDP cells were determined by western blot analysis. (E and F) The migration and invasion of A549 and A549/DDP cells were examined by Transwell assay. "P $<0.05$ vs. respective control. LUCAT1, lung cancer-associated transcript 1; DDP, cisplatin; NSCLC, non-small cell lung cancer.

of LUCAT1 in DDP resistance in NSCLC, the expression of LUCAT1 in DDP-resistant NSCLC tissues and cells was first determined. The results of RT-qPCR revealed that LUCAT1 was markedly upregulated in NSCLC tissues (DDP-resistant NSCLC tissues and DDP-sensitive NSCLC tissues) and cells
(A549 cells and A549/DDP cells) compared to that in normal tissues and cells; furthermore, LUCAT1 expression was higher in DDP-resistant NSCLC tissues and cells when compared to DDP-sensitive NSCLC tissues and cells (Fig. 1A and B). In addition, the $\mathrm{IC}_{50}$ value of cisplatin evidently increased 
A

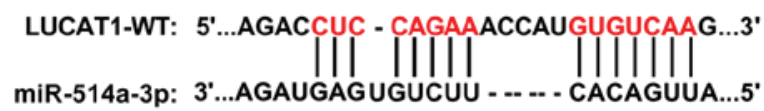

LUCAT1-MUT: 5'...AGACGAG - GUCUUACCAUCACAGUUG...3'
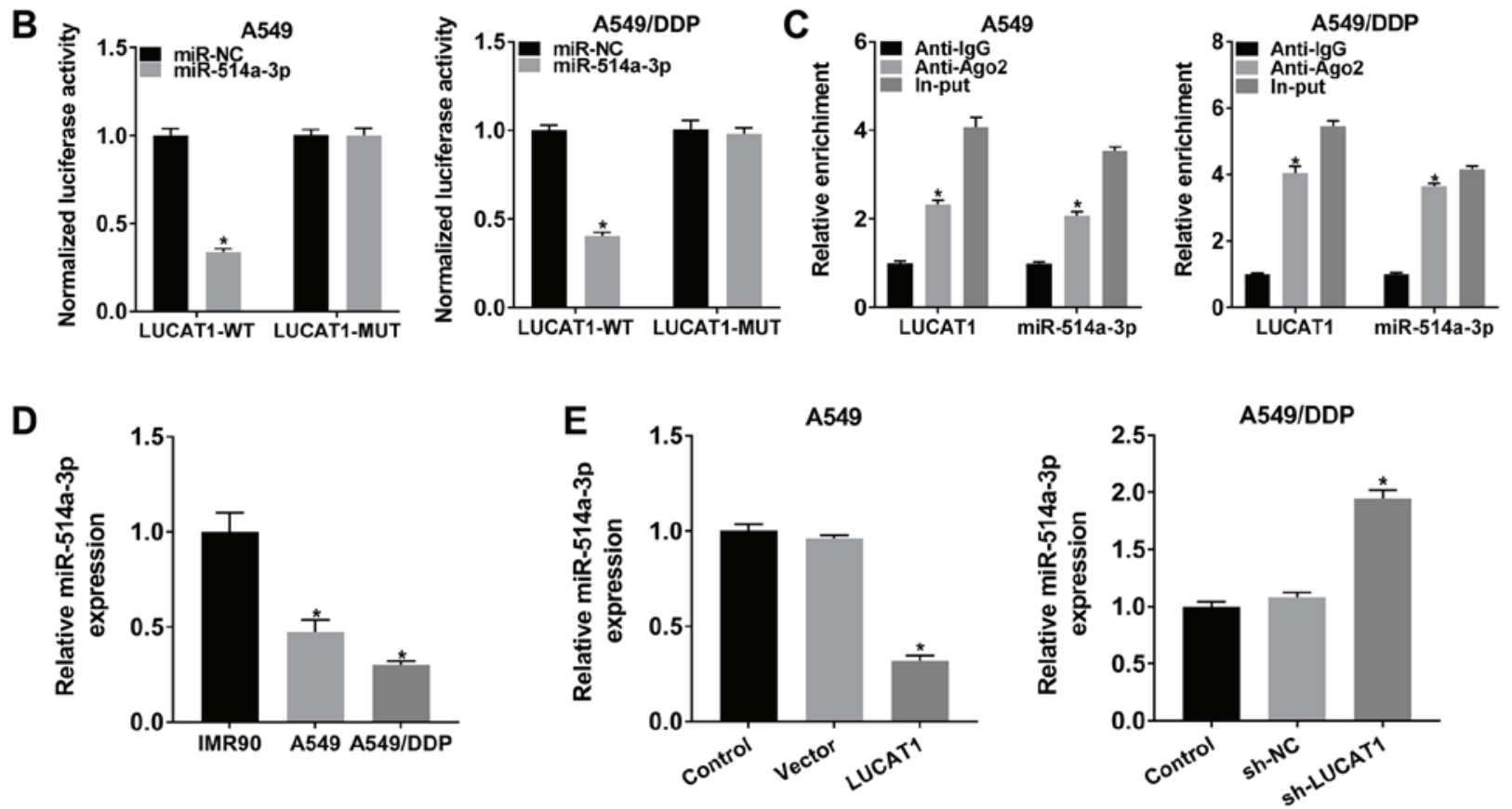

Figure 3. LUCAT1 directly binds to miR-514a-3p and negatively alters the expression of miR-514a-3p. (A) The predicted binding sites between LUCAT1 and miR-514a-3p are presented. (B) Dual-luciferase reporter assay was performed to detect the luciferase activity in LUCAT1-WT or LUCAT1-MUT and miR-NC or miR-514a-3p co-transfected A549 and A549/DDP cells. (C) The enrichment of LUCAT1 and miR-514a-3p was analyzed by RT-qPCR after RIP assay in A549 and A549/DDP cells. (D) The level of miR-514a-3p in IMR90, A549 and A549/DDP cells was measured by RT-qPCR. (E) A549 cells were transfected with Vector or LUCAT1, A549/DDP cells were transfected with sh-NC or sh-LUCAT1, and untransfected A549 cells and A549/DDP cells were used as the control groups. The expression of miR-514a-3p was then examined by RT-qPCR. "P<0.05 vs. respective control. LUCAT1, lung cancer-associated transcript 1; DDP, cisplatin; NSCLC, non-small cell lung cancer.

in the A549/DDP compared to the A549 cells, indicating that cisplatin resistance was established in the A549/DDP cells (Fig. 1C). Moreover, the cells were treated with various concentrations of cisplatin for $24 \mathrm{~h}$ and CCK-8 assay was then performed. The results demonstrated that the viability of the A549/DDP cells was increased compared to that of the A549 cells (Fig. 1D). Cells treated with $2 \mu \mathrm{g} / \mathrm{ml}$ cisplatin were used in the subsequent experiments. All these data indicated that LUCAT1 may play a role in the regulation of DDP resistance in NSCLC.

LUCAT1 enhances cisplatin resistance by inhibiting the apoptosis, and promoting the viability, autophagy, migration and invasion in NSCLC cells. In view of the fact that LUCAT1 expression was higher in the A549/DDP cells compared to the A549 cells, LUCAT1 overexpression vector was transfected into A549 cells and sh-LUCAT1 was transfected into A549/DDP cells to explore the functional role of LUCAT1 in the regulation of the cisplatin resistance of NSCLC cells. As shown in Fig. 2A, LUCAT1 overexpression led to a marked increase in LUCAT1 expression in the A549 cells and sh-LUCAT1 transfection led to a marked decrease in LUCAT1 expression in the A549/DDP cells. CCK- 8 assay revealed that the viability of the A549 cells was promoted following the overexpression of LUCAT1 and was suppressed in the A549/DDP cells following the knockdown of LUCAT1 (Fig. 2B). Cisplatin treatment induced the apoptosis of the A549 cells and A549/DDP cells; however, the apoptosis of the A549 cells was suppressed following the overexpression of LUCAT1 and that of the A549/DDP cells was promoted following the knockdown of LUCAT1, as indicated by flow cytometric analysis (Fig. 2C).

If soluble p62 is decreased, insoluble p62 is stable, and the ratio of LC3-II/LC3-I is increased, indicating the activation of autophagy. Thus, the levels of autophagy-related proteins in the A549 cells and A549/DDP cells were determined by western blot analysis. It was observed that cisplatin treatment increased the ratio of LC3-II/LC3-I and decreased the level of soluble p62 in the A549 cells and A549/DDP cells; moreover, the elevated expression of LUCAT1 further enhanced the LC3-II/LC3-I ratio and decreased the soluble p62 level in the A549 cells; however, the silencing of LUCAT1 decreased the LC3-II/LC3-I ratio and increased the soluble p62 level in the A549/DDP cells (Fig. 2D). However, the level of insoluble p62 was not altered when the A549 and A549/DDP cells were subjected to the above-mentioned treatments (Fig. 2D). When autophagy was enhanced, the ratio of LC3-II/LC3-I was increased and the level of p62 was decreased. 

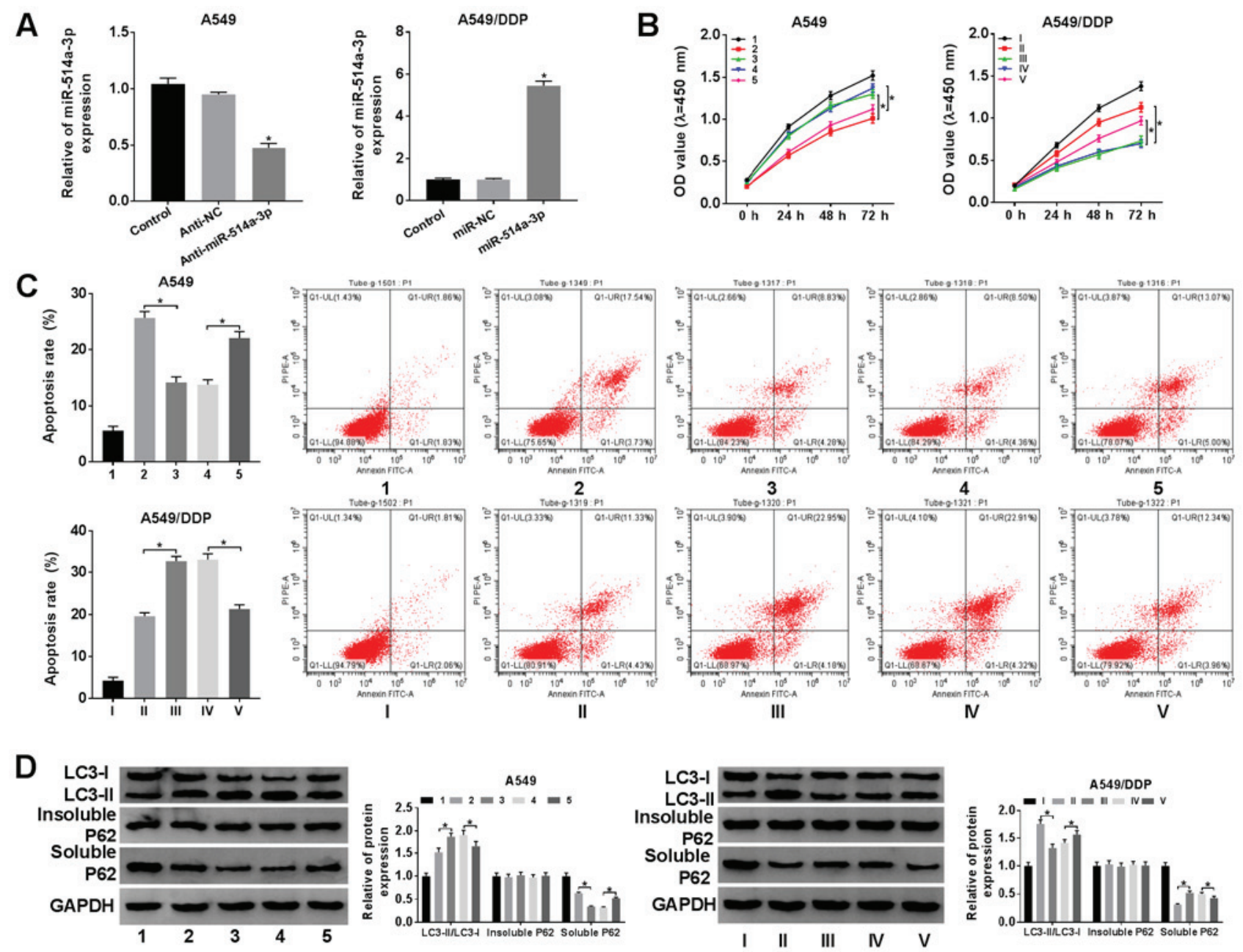

E
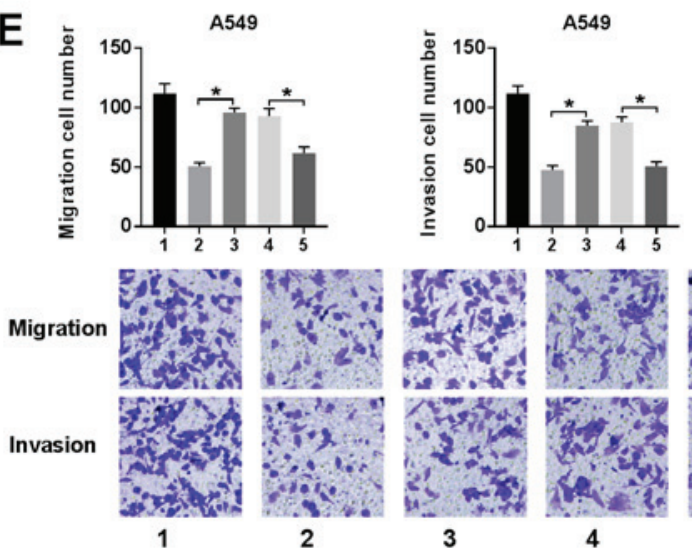
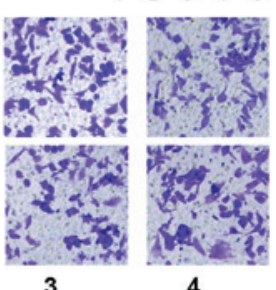

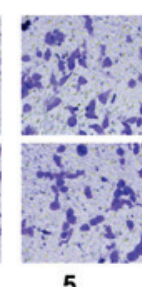

$\mathbf{F}$
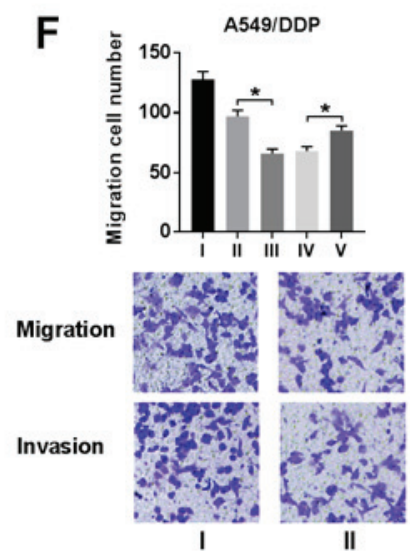
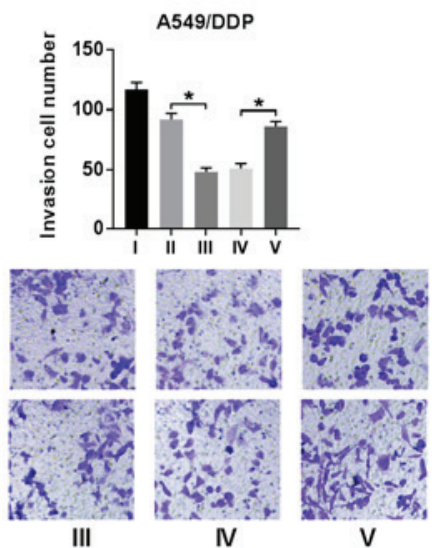

Figure 4. LUCAT1 overexpression reverses the inhibitory effects of miR-514a-3p overexpression on cisplatin resistance in NSCLC cells. (A) A549 cells were transfected with Anti-NC or Anti-miR-514a-3p, A549/DDP cells were transfected with miR-NC or miR-514a-3p, and untransfected A549 cells and A549/DDP cells were used as the control groups. The the level of miR-514a-3p was then detected by RT-qPCR. (B-F) The A549 cells were assigned to the control (1), cisplatin + Anti-NC (2), cisplatin + Anti-miR-514a-3p (3), cisplatin + Anti-miR-514a-3p + sh-NC (4) and cisplatin + Anti-miR-514a-3p + sh-LUCAT1 (5) groups, and the A549/DDP cells were assigned to the control (I), cisplatin + miR-NC (II), cisplatin + miR-514a-3p (III), cisplatin + miR-514a-3p + Vector (IV) and cisplatin + miR-514a-3p + LUCAT1 (V) groups. (B) The viability of the A549 and A549/DDP cells was analyzed by CCK-8 assay. (C) The apoptosis of the A549 and A549/DDP cells was evaluated by flow cytometric analysis. (D) The ratio of LC3-II/LC3-I and the levels of insoluble p62 and soluble p62 were examined by western blot analysis. ( $\mathrm{E}$ and F) The migration and invasion of A549 and A549/DDP cells were analyzed by Transwell assay. ${ }^{*} \mathrm{P}<0.05$ vs. respective control. LUCAT1, lung cancer-associated transcript 1; DDP, cisplatin; NSCLC, non-small cell lung cancer.

The results of Transwell assay indicated that cisplatin treatment markedly suppressed the migration and invasion of the A549 and A549/DDP cells, whereas these effects were abrogated in the A549 cells following LUCAT1 overexpression.
In addition, the migration and invasion of the A549/DDP cells were further suppressed following transfection with sh-LUCAT1, indicating that LUCAT1 knockdown counteracted the resistance of NSCLC cells to cisplatin, at least to a 
A

ULK1-3'UTR-WT: 5'...GAUACCCACCCAGCUUUGUGAAU....'
miR-514a-3p:

ULK1-3'UTR-MUT: 5'...GAAUGCGUCCGUCCUUACAGUUU...3'
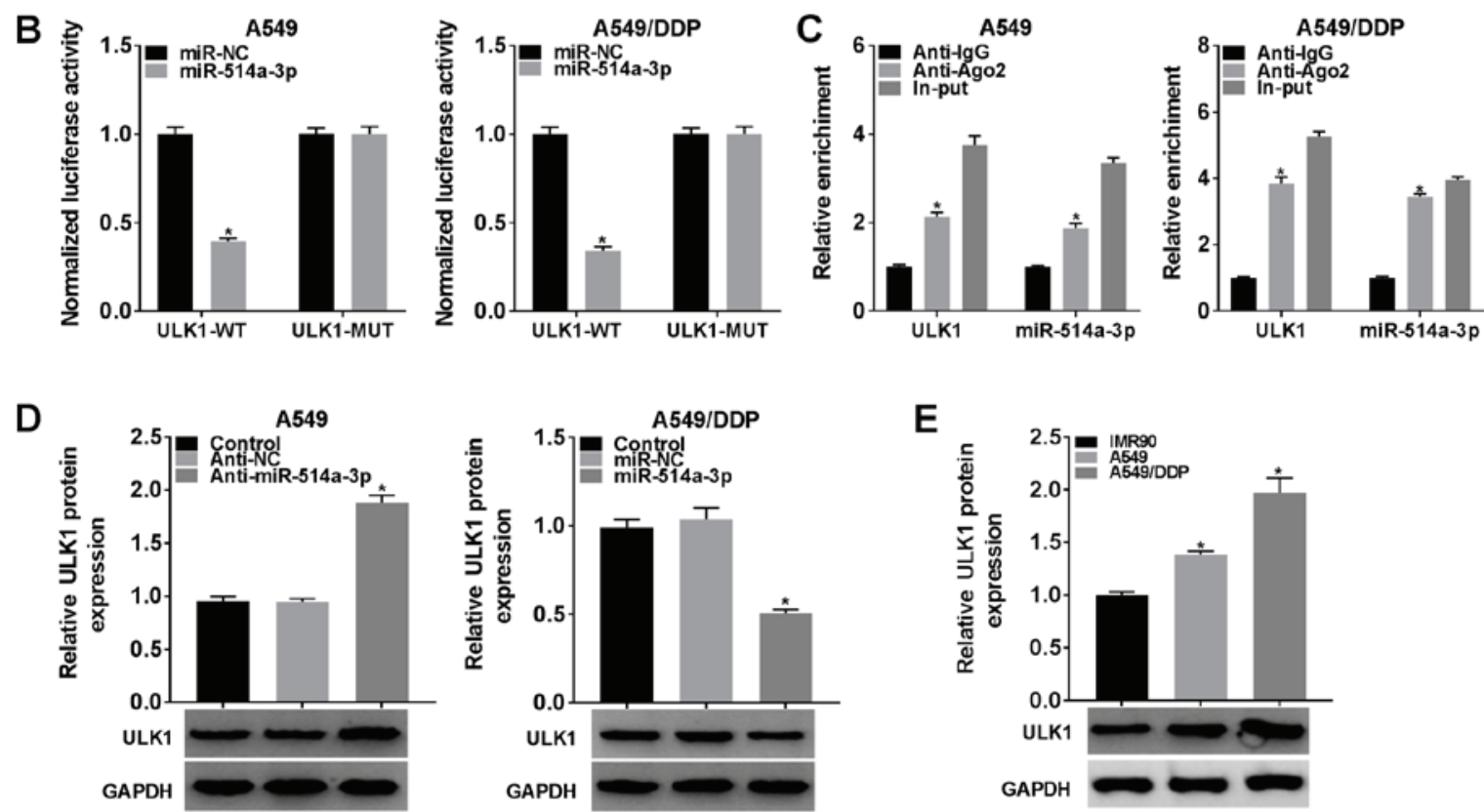

Figure 5. miR-514a-3p directly targets ULK1 to modulate ULK1 expression. (A) The potential binding sites between miR-514a-3p and ULK1 were predicted by StarBase 3.0. (B) Dual-luciferase reporter assay was conducted to confirm the association between miR-514a-3p and ULK1. (C) The enrichment of ULK1 and miR-514a-3p was analyzed by RT-qPCR following RIP assay. (D) The protein level of ULK1 in A549 cells transfected with Anti-NC or Anti-miR-514a-3p and in A549/DDP cells transfected with miR-NC or miR-514a-3p was determined by western blot analysis. (E) The protein level of ULK1 in IMR90, A549 and A549/DDP cells was detected by western blot analysis. "P<0.05 vs. the respective control. ULK1, uncoordinated-51-like kinase 1; DDP, cisplatin.

certain extent (Fig. 2E and F). Thus, these results demonstrate that LUCAT1 can promote cisplatin resistance by suppressing the apoptosis, and promoting the viability, autophagy and metastasis of NSCLC cells.

LUCAT1 negatively regulates miR-514a-3p expression by directly targeting $m i R-514 a-3 p$. To determine the underlying mechanisms of LUCAT1, the online software StarBase 3.0 was searched and it was found that miR-514a-3p was a target of LUCAT1 (Fig. 3A). To verify this, a dual-luciferase reporter assay and RIP assay were carried out. As shown in Fig. 3B, co-transfection with LUCAT1-WT and miR-514a-3p resulted in an evident inhibition of the luciferase activity in the A549 cells and A549/DDP cells compared with that in the cells co-transfected with LUCAT1-WT and miR-NC; however, the luciferase activity was not affected in the LUCAT1-MUT group.

To act as miRNA sponges, lncRNAs need to be predominantly enriched in the cytoplasm and effectively accessible to RNA-induced silencing complex (RISC) $(21,22)$. In the present study, RIP assay revealed that LUCAT1 and miR-514a-3p were enriched in Anti-Ago2 immunoprecipitation complexes compared with Anti-lgG immunoprecipitates in the A549 cells and A549/DDP cells, indicating that LUCAT1 and miR-514a-3p existed in the RISC, further confirming the association between LUCAT1 and miR-514a-3p (Fig. 3C). In addition, as was expected, the expression of miR-514a-3p was decreased in the A549 cells compared to the IMR90 cells; moreover, miR-514a-3p expression was lower in the A549/DDP cells than in the A549 cells (Fig. 3D).
Furthermore, the level of miR-514a-3p in the A549 cells transfected with the LUCAT1 overexpression vector and in the sh-LUCAT1-transfected A549/DDP cells was examined by RT-qPCR. The results revealed that the miR-514a-3p level was markedly decreased in the A549 cells transfected with the LUCAT1 overexpression vector, and was increased in the A549/DDP cells transfected with sh-LUCAT1 (Fig. 3E). Taken together, these results demonstrate that LUCAT1 functions as an miR-514a-3p sponge to alter miR-514a-3p expression in A549 and A549/DDP cells.

The inhibitory effect of miR-514a-3p on cisplatin resistance of NSCLC cells was reversed by LUCAT1. Since LUCAT1 could directly interact with miR-514a-3p and negatively regulate $\mathrm{miR}-514 \mathrm{a}-3 \mathrm{p}$ expression, we hypothesized that LUCAT1 could improve cisplatin resistance via targeting miR-514a-3p in NSCLC. As shown in Fig. 4A, transfection with anti-miR-514a-3p led to a marked decrease in miR-514a-3p expression in the A549 cells, and transfection with miR-514a-3p mimics led to an evident increase in miR-514a-3p expression in the A549/DDP cells. Subsequently, the A549 cells were treated with cisplatin + Anti-NC, cisplatin + Anti-miR-514a-3p, cisplatin + Anti-miR-514a-3p + sh-NC or cisplatin + Anti-miR-514a-3p + sh-LUCAT1, and the A549/DDP cells were treated with cisplatin + miR-NC, cisplatin + miR-514a-3p, cisplatin + miR-514a-3p + Vector or cisplatin + miR-514a-3p + LUCAT1. CCK-8 assay revealed that cell viability was markedly promoted by miR-514a-3p 

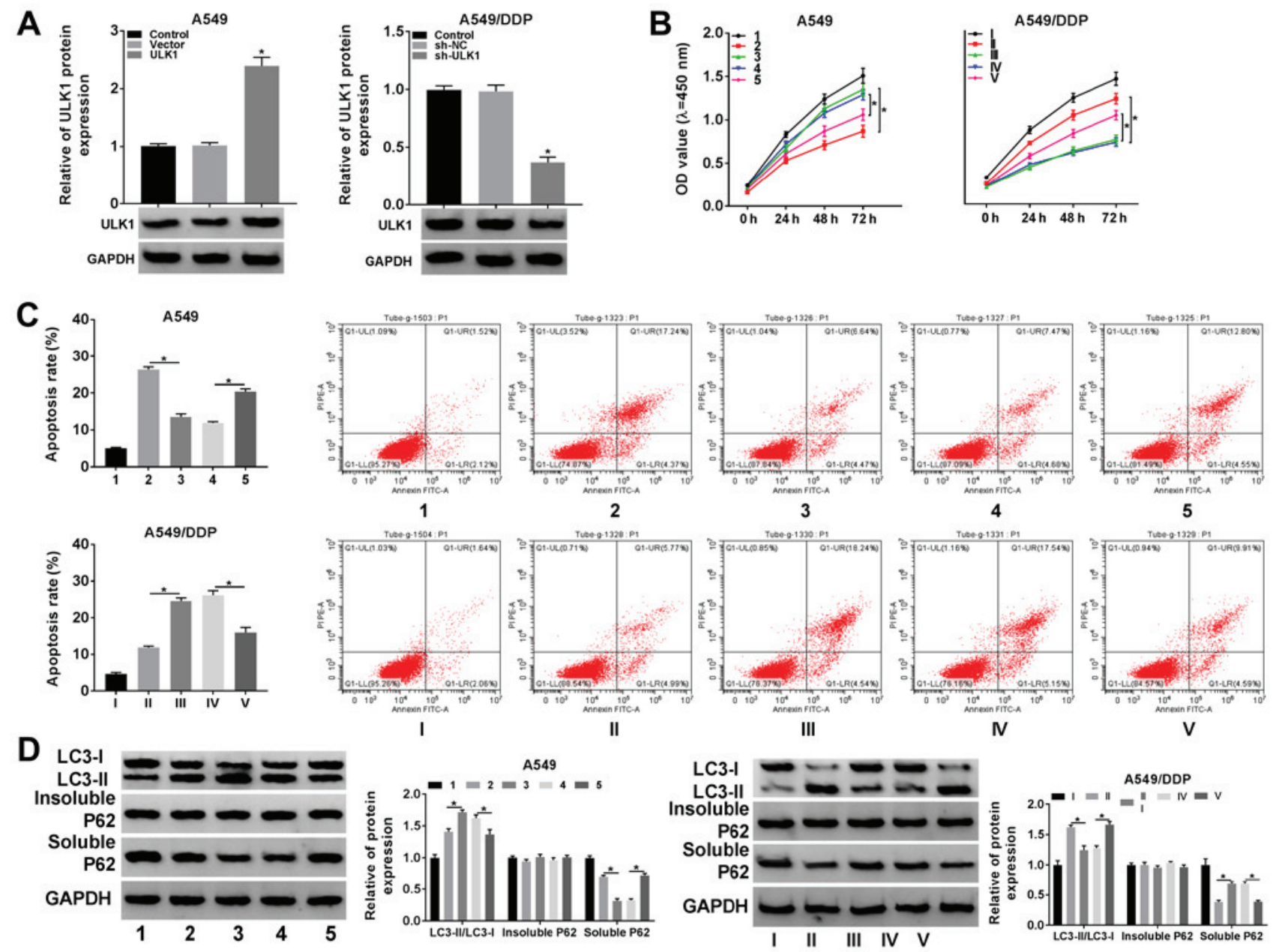

E
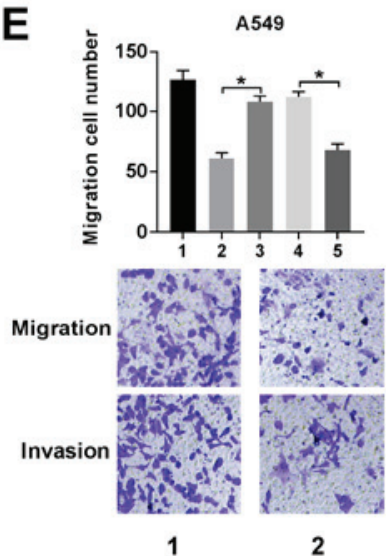
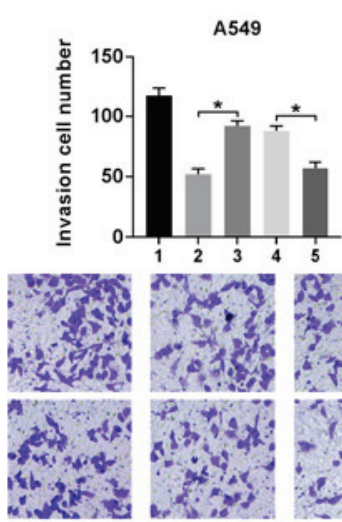

3

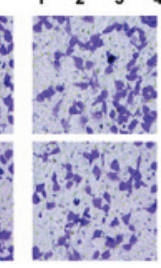

4
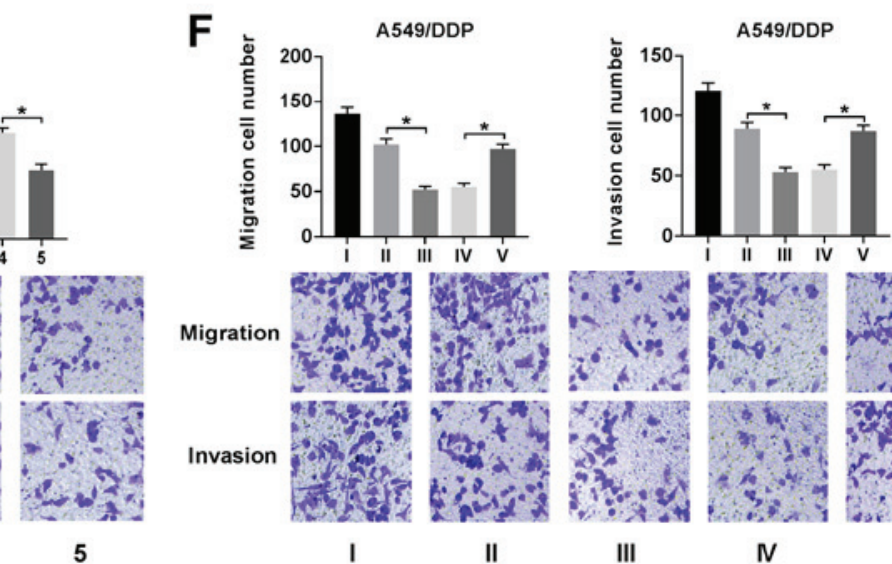

I

II

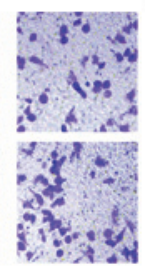

III

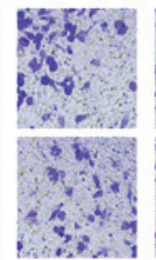

N

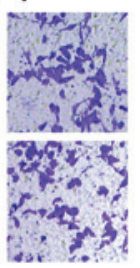

V

Figure 6. miR-514a-3p inhibition abolishes the effects of ULK1 knockdown on cisplatin resistance in NSCLC cells. (A) A549 cells were transfected with Vector or ULK1, A549/DDP cells were transfected with sh-NC or sh-ULK1, and untransfected A549 cells and A549/DDP cells were used as the control groups; the protein level of ULK1 was measured by western blot analysis. (C-F) A549 cells were assigned to the control (1), cisplatin + Vector (2), cisplatin + ULK1 (3), cisplatin + ULK1 + miR-NC (4) and cisplatin + ULK1 + miR-514a-3p (5) groups; the A549/DDP cells were assigned to the control (I), cisplatin + sh-NC (II), cisplatin + sh-ULK1 (III), cisplatin + sh-ULK1 + Anti-NC (IV) and cisplatin + sh-ULK1 + Anti-miR-514a-3p (V) groups. (B) The viability of the A549 and A549/DDP cells was examined by CCK-8 assay. (C) The apoptosis of the A549 and A549/DDP cells was assessed via flow cytometric analysis. (D) The ratio of LC3-II/LC3-I and the levels of insoluble p62 and soluble p62 were measured using western blot analysis. (E and F) The migration and invasion of A549 and A549/DDP cells were determined by Transwell assay. "P<0.05 vs. the respective control. ULK1, uncoordinated-51-like kinase 1; DDP, cisplatin; NSCLC, non-small cell lung cancer.

inhibition and LUCAT1 knockdown reversed this effect in the A549 cells. Moreover, cell viability was evidently suppressed following the upregulation of miR-514a-3p and LUCAT1 overexpression reversed this effect in the A549/DDP cells (Fig. 4B).
The results of flow cytometric analysis demonstrated that Anti-miR-514a-3p suppressed the apoptosis of the A549 cells and sh-LUCAT1 abolished this suppression. Moreover, miR-514a-3p evidently accelerated A549/DDP cell apoptosis and LUCAT1 overexpression effectively attenuated this 
A

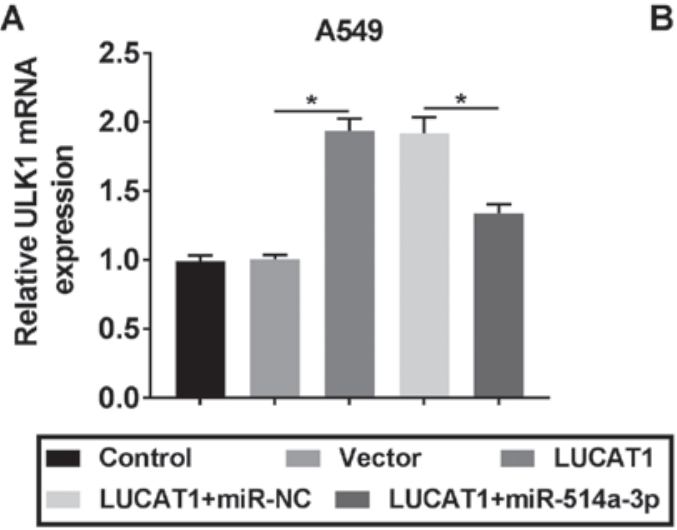

C

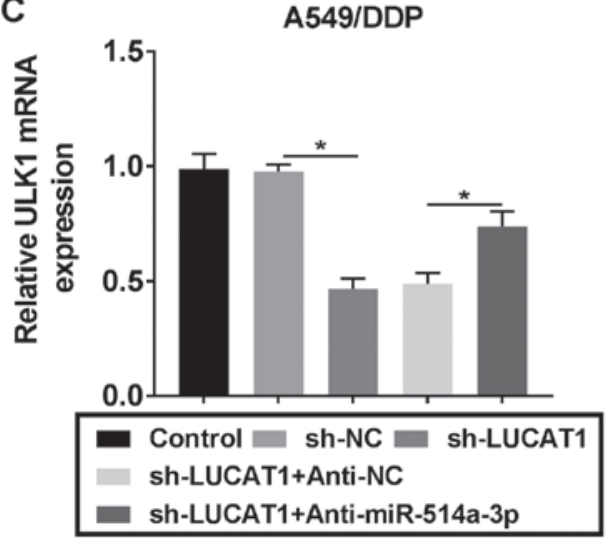

B

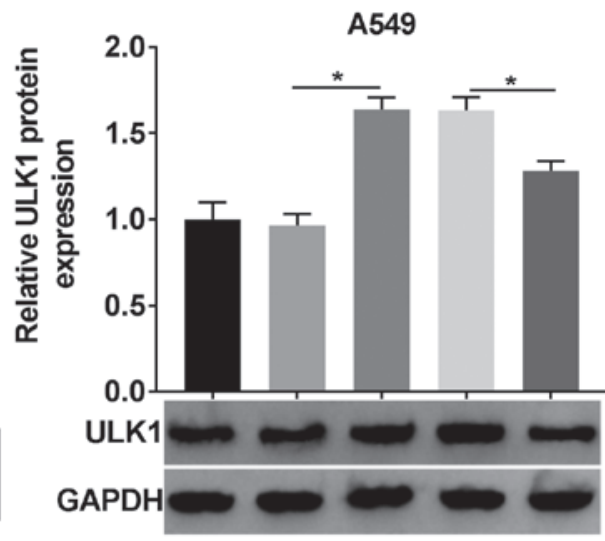

D

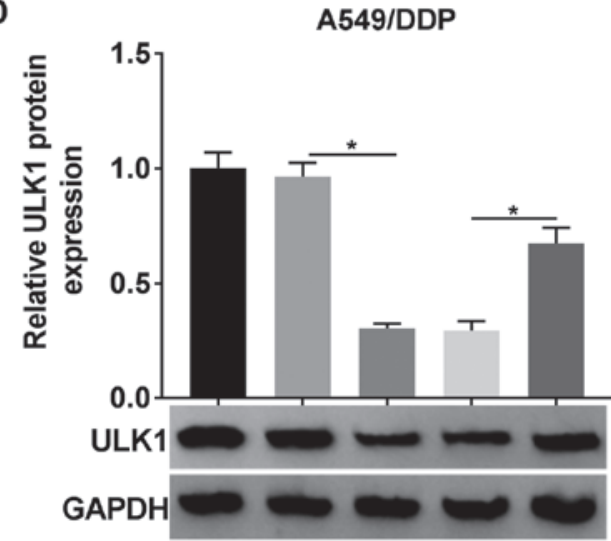

Figure 7. LUCAT1 alters ULK1 expression via acting as a sponge of miR-514a-3p in NSCLC cells. (A and B) A549 cells were transfected with Vector, LUCAT1, LUCAT1 + miR-NC or LUCAT1 + miR-514a-3p and then the mRNA and protein levels of ULK1 were examined by RT-qPCR and western blot analysis, respectively. (C and D) A549/DDP cells were transfected with sh-NC, sh-LUCAT1, sh-LUCAT1 + Anti-NC or sh-LUCAT1 + Anti-miR-514a-3p and the mRNA and protein levels of ULK1 were then examined by RT-qPCR and western blot analysis, respectively. ${ }^{*} \mathrm{P}<0.05$ vs. the respective control. LUCAT1, lung cancer-associated transcript 1; ULK1, uncoordinated-51-like kinase 1; DDP, cisplatin; NSCLC, non-small cell lung cancer.

effect (Fig. 4C). As demonstrated by western blot analysis, the LC3-II/LC3-I ratio was increased and the soluble p62 level was decreased in the A549 cells transfected with Anti-miR-514a-3p; these effects were partly reversed after the cells were transfected with sh-LUCAT1; however, no change was observed in the insoluble p62 expression level (Fig. 4D, upper panels). In the A549/DDP cells, miR-514a-3p overexpression markedly inhibited the LC3-II/LC3-I ratio and promoted the soluble p62 level, and LUCAT1 overexpression abolished these effects; however, the insoluble p62 level was not altered after these treatments (Fig. 4D, lower panels).

Transwell assay also revealed that A549 cell migration and invasion were promoted by miR-514a-3p inhibition, while these effects were abolished by LUCAT1 knockdown (Fig. 4E). Transwell assay also revealed that A549/DDP cell migration and invasion were suppressed by miR-514a-3p overexpression, whereas the overexpression of LUCAT attenuated these effects (Fig. 4F). These, these findings demonstrate that LUCAT1 promotes cisplatin resistance by targeting miR-514a-3p in NSCLC cells.

miR-514a-3p negatively modulates ULK1 expression by direct interaction. Based on the above-mentioned data, StarBase 3.0 was further searched and it was found that ULK1 was a target gene of miR-514a-3p; their binding sites are shown in Fig. 5A. As suggested by dual-luciferase reporter assay, the luciferase activity was markedly suppressed in the A549 cells and A549/DDP cells co-transfected with miR-514a-3p and ULK1-WT compared with that in the cells co-transfected with miR-NC and ULK1-WT, whereas the luciferase activity was not altered in the ULK1-MUT group (Fig. 5B).

RIP assay revealed that the enrichment of ULK1 and miR-514a-3p in the A549 cells and A549/DDP cells was markedly increased in the Anti-Ago2 RIP group compared with the Anti-IgG RIP group, suggesting that ULK1 could bind to the RISC consisting miR-514a-3p (Fig. 5C). Subsequently, Anti-NC or Anti-miR-514a-3p were transfected into the A549 cells, and miR-NC or miR-514a-3p were transfected into the A549/DDP cells to further investigate the association between ULK1 and miR-514a-3p. As shown in Fig. 5D, Anti-miR-514a-3p transfection resulted in a marked increase in the ULK1 protein level in the A549 cells, and transfection with miR-514a-3p mimic led to an effective decrease in ULK1 protein expression in the A549/DDP cells. In addition, the protein level of ULK1 in THE IMR90, A549 and A549/DDP cells was determined. As was expected, the protein level of ULK1 was increased in the A549 cells compared with the IMR90 cells, and ULK1 expression in the A549/DDP cells was higher than that in 
A
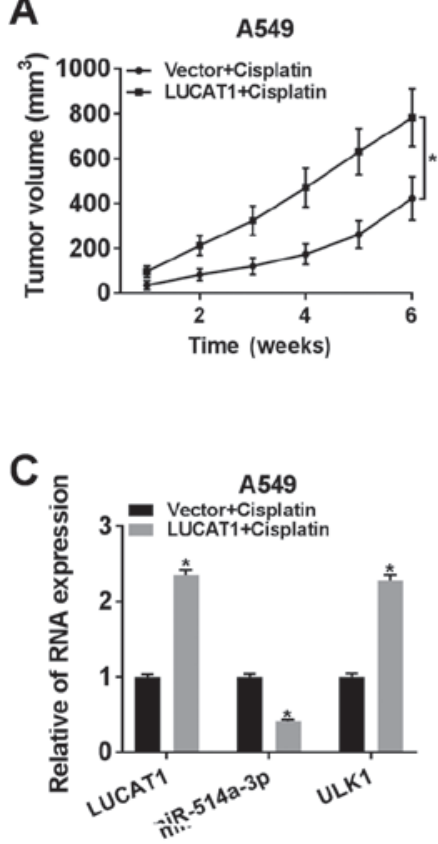

A549
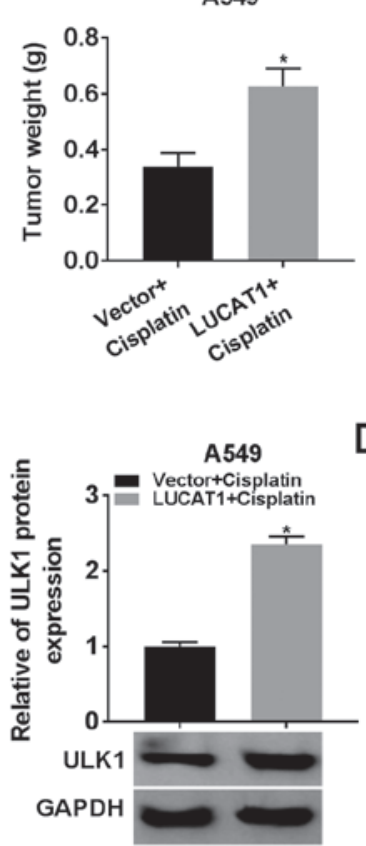

B

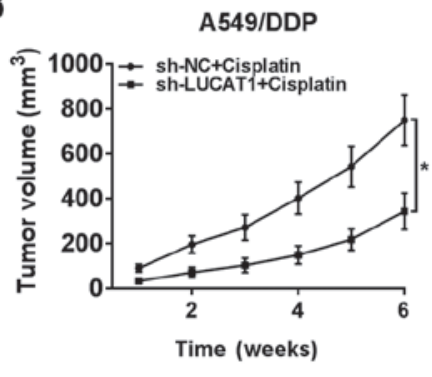

D

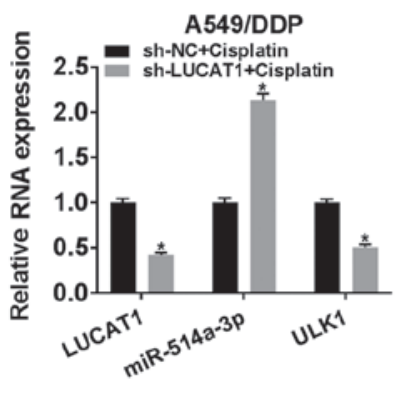

A549/DDP
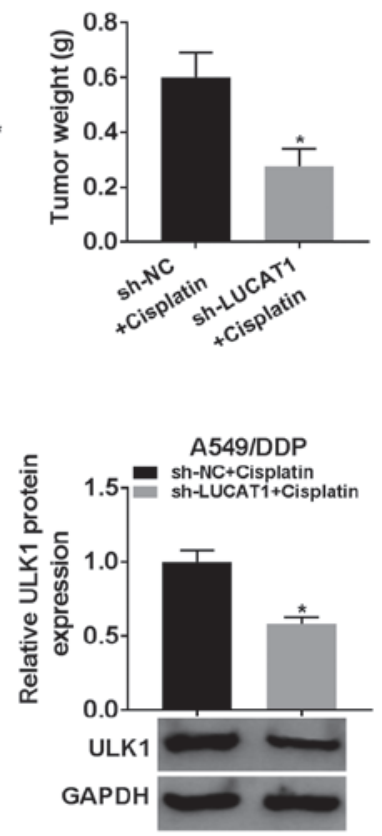

Figure 8. LUCAT1 overexpression promotes tumor growth and cisplatin resistance in vivo. (A and B) Tumor volume was measured each week and tumor weight was measured after 6 weeks. (C and D) The expression levels of LUCAT1, miR-514a-3p and ULK1 mRNA in tumor samples were detected by RT-qPCR, and the protein level of ULK1 was determined by western blot analysis. " $\mathrm{P}<0.05$ vs. the respective control. LUCAT1, lung cancer-associated transcript 1 ; ULK1, uncoordinated-51-like kinase 1; DDP, cisplatin.

the A549 cells (Fig. 5E). All these data indicated that ULK1 was negatively regulated by miR-514a-3p in NSCLC cells.

Silencing of miR-514a-3p attenuates the inhibitory effect of ULK1 knockdown on the resistance of NSCLC cells to cisplatin. To reveal the function of miR-514a-3p and ULK1 in regulating the resistance of NSCLC cells to cisplatin, ULK1 or Vector were transfected into the A549 cells, and sh-NC or sh-ULK1 were transfected into the A549/DDP cells. The results of western blot analysis revealed that the protein expression of ULK1 was markedly increased in the A549 cells following transfection with ULK1 overexpression vector, and was notably decreased in the A549/DDP cells following transfection with sh-ULK1 (Fig. 6A). It was then demonstrated that ULK1 overexpression markedly enhanced the viability of the A549 cells and this enhancement was suppressed by miR-514a-3p overexpression; moreover, ULK1 knockdown evidently suppressed the viability of the A549/DDP cells and this suppression was attenuated by transfection with Anti-miR-514a-3p, as illustrated by CCK-8 assay (Fig. 6B).

In addition, the results of flow cytometric analysis indicated that the decreased cell apoptosis induced by ULK1 overexpression was evidently increased in the A549 cells following the overexpression of miR-514a-3p, and the increased cell apoptosis mediated by sh-ULK1 was significantly inhibited in the A549/DDP cells following the downregulation of miR-514a-3p (Fig. 6C). It was also demonstrated that the ratio of LC3-II/LC3-I was elevated and the protein level of soluble p62 was decreased following the overexpression of ULK1 in the A549 cells, and these effects were restored following miR-514a-3p upregulation; however, the insoluble p62 level was not altered following these treatments, indicating that the enhancement of autophagy caused by ULK1 was reversed by miR-514a-3p overexpression (Fig. 6D, upper panels). In the A549/DDP cells, ULK1 downregulation markedly decreased the ratio of LC3-II/LC3-I and induced the level of soluble p62, whereas the inhibition of miR-514a-3p partly restored these effects; however, the level of insoluble p62 was stable following these treatments (Fig. 6D, lower panels).

Furthermore, Transwell assay indicated that ULK1 overexpression promoted the migration and invasion of the A549 cells, while the overexpression of $\mathrm{miR}-514 \mathrm{a}-3 \mathrm{p}$ reversed these effects (Fig. 6E). The silencing of ULK1 markedly impeded the migration and invasion of the A549/DDP cells, while miR-514a-3p inhibition reversed these effects (Fig. 6F). Collectively, these data indicated tht miR-514a-3p bound to ULK1 to regulate cisplatin resistance in NSCLC cells.

LUCAT1 positively regulates ULK1 expression by sponging miR-514a-3p in NSCLC cells. In order to determine the association between LUCAT1, miR-514a-3p and ULK1, the A549 cells were assigned to the control, Vector, LUCAT1, LUCAT1 + miR-NC and LUCAT1 + miR-514a-3p groups, and the A549/DDP cells were assigned to the control, sh-NC, sh-LUCAT1, sh-LUCAT1 + Anti-NC and sh-LUCAT1 + Anti-miR-514a-3p groups. It was found that the mRNA and protein levels of ULK1 were increased in the A549 cells following the overexpression of LUCAT1, while miR-514a-3p upregulation partially suppressed this increase (Fig. 7A and B). The knockdown of LUCAT1 decreased the mRNA and protein levels of ULK1 in the A549/DDP cells, whereas miR-514a-3p inhibition abolished these effects (Fig. 7C and D). Thus, it was demonstrated that LUCAT1 upregulated the expression of ULK1 by targeting miR-514a-3p in NSCLC cells. 
LUCAT1 knockdown suppresses tumorigenesis and cisplatin resistance in vivo. To investigate the role of LUCAT1 in vivo, A549 cells transfected with Vector or LUCAT1 and A549/DDP cells transfected with sh-NC or sh-LUCAT1 were injected into mice, and the tumor volume was then examined weekly, and tumor weight was examined after 6 weeks. The data indicated that LUCAT1 upregulation evidently promoted tumor growth and tumor weight compared to the Vector group (Fig. 8A). The downregulation of LUCAT1 notably suppressed tumor growth and tumor weight compared with the sh-NC group (Fig. 8B). Furthermore, the levels of LUCAT1, miR-514a-3p and ULK1 in the collected tumor tissues were measured. The results revealed that the expression levels of LUCAT1, ULK1 mRNA and ULK1 protein were markedly upregulated, and the expression level of miR-514a-3p was notably downregulated in the tumor tissues from the LUCAT1 group; however, the opposite results were observed in the tumor tissues from the sh-LUCAT1 group (Fig. 8C and D). These data suggested that the knockdown of LUCAT1 inhibited tumor growth and cisplatin resistance in vivo.

\section{Discussion}

Currently, an increasing number of researchers are focusing on the effects of lncRNAs on tumor development and drug resistance in human cancers $(23,24)$. In the present study, the effect of LUCAT1 on cisplatin resistance in NSCLC was investigated. It was found that the LUCAT1 level was elevated in the A549/DDP cells, and LUCAT1 knockdown enhanced the sensitivity of A549/DDP cells to cisplatin by regulating the miR-514a-3p/ULK1 axis.

Zheng et al demonstrated that the LUCAT1 level was elevated in cisplatin-resistant ovarian cancer cells (25). Han and Shi suggested that LUCAT1 was highly expressed in methotrexate-resistant osteosarcoma (OS) cells, and the inhibition of LUCAT1 markedly hampered methotrexate resistance, cell growth and metastasis in OS (26). Furthermore, Wang et al indicated that LUCAT1 expression was higher in A549/DDP cells than in A549 cells; moreover, LUCAT1 elevation suppressed cell apoptosis and cisplatin sensitivity in NSCLC cells (27). Consistently, in the present study, it was observed that the LUCAT1 level was markedly increased in DDP-resistant NSCLC tissues and cells. The knockdown of LUCAT1 evidently suppressed the viability and motility, and induced the apoptosis of DDP-resistant NSCLC cells. Autophagy is a process of cell self-degradation used to remove damaged or redundant proteins and organelles, and can be observed in a number of physiological and pathological processes (28). It has been reported that autophagy is associated with drug resistance in malignant tumors. For example, Yan et al demonstrated that HOTAIR silencing relieved drug resistance by suppressing the activation of autophagy in NSCLC (29). Xiong et al elucidated that HULC improved the chemoresistance of HCC cells by activating autophagy (30). To the best of our knowledge, the present study was the first to examine the effects of LUCAT1 on the protein levels of autophagy regulators and it was found that LUCAT1 knockdown decreased the LC3-II/LC3-I ratio and increased the p62 level in A549/DDP cells, suggesting that autophagy was suppressed. These data suggest that LUCAT1 plays a positive role in cisplatin resistance in NSCLC.
Subsequently, the present study explored the underlying mechanisms of LUCAT1 in the regulation of the drug sensitivity of NSCLC cells. LUCAT1 was identified to function as a sponge of miR-514a-3p and the miR-514a-3p level was decreased in A549/DDP cells. miR-514a-3p has been confirmed to be weakly expressed and to play a tumor-suppressive role in renal cell carcinoma by suppressing cell growth and inducing cell apoptosis (31). Here, we determined the role of miR-514a-3p in drug resistance for the first time. The present study found that the upregulation of miR-514a-3p suppressed cell viability and metastasis, facilitated cell apoptosis and inactivated autophagy in cisplatin-resistant NSCLC cells, whereas these effects were partly abolished following LUCAT1 overexpression.

Moreover, ULK1 was confirmed to be a target of miR-514a-3p. ULK1 is a regulator of autophagy and plays a role part in the regulation of drug resistance in human tumors (32). For example, Tang et al proved that ULK1 knockdown suppressed cell growth and cisplatin resistance by modulating the apoptosis and autophagy of NSCLC cells (33). Yang et al suggested that HOTAIR downregulation improved crizotinib sensitivity by hindering cell viability and autophagy and accelerating cell apoptosis in NSCLC through decreasing ULK1 expression (29). Consistently, the present study observed that the ULK1 level was decreased in A549/DDP cells, and ULK1 deficiency inhibited cisplatin resistance by inhibiting cell viability and metastasis, promoting cell apoptosis and blocking autophagy in cisplatin-resistant NSCLC cells, whereas inhibitors of miR-514a-3p abolished this inhibition.

However, the present study also contains certain shortcomings. For example, TUNEL staining assay was not performed to examine the cell apoptotic ability. Moreover, immunocytochemistry and immunofluorescence microscopy were not conducted to examine the intracellular localization of LC3-II and the localization of different forms of p62 in cells. The authors thus aim to conduct these experiments in the future.

In conclusion, the present study demonstrated that the LUCAT1 level was evidently increased in DDP-resistant NSCLC cells. LUCAT1 silencing enhanced cisplatin sensitivity by inducing cell apoptosis, and suppressing autophagy and cell metastasis in NSCLC. Moreover, LUCAT1 regulated the sensitivity of NSCLC cells to cisplatin by upregulating ULK1 via sponging miR-514a-3p. These findings provide a novel regulatory network of the LUCAT1/miR-514a-3p/ULK1 axis in regulating the chemoresistance of NSCLC. These findings suggest that LUCAT1 may be a potential target which may be used to counteract the resistance of NSCLC to DDP.

\section{Acknowledgements}

Not applicable.

\section{Funding}

No funding was received.

\section{Availability of data and materials}

The analyzed data sets generated during the study are available from the corresponding author on reasonable request. 


\section{Authors' contributions}

QS conceived the study, and ZX and SX designed the study. QMS was involved in data collection. ZX performed the statistical analysis and prepared the figures. QS drafted the manuscript. SX contributed substantially to the revision of the manuscript. All authors read and approved the final manuscript.

\section{Ethics approval and consent to participate}

The study protocol was approved by the Ethics Committee of the First Hospital of China Medical University. Written informed consent forms were signed by the patients.

\section{Patient consent for publication}

Not applicable.

\section{Competing interests}

The authors declare that they have no competing interests.

\section{References}

1. Bray F, Ferlay J, Soerjomataram I, Siegel RL, Torre LA and Jemal A: Global cancer statistics 2018: GLOBOCAN estimates of incidence and mortality worldwide for 36 cancers in 185 countries. CA Cancer J Clin 68: 394-424, 2018.

2. Fitzmaurice C, Akinyemiju TF, Al Lami FH, Alam T, Alizadeh-Navaei R, Allen C, Alsharif U, Alvis-Guzman N, Amini E and Anderson BO: Global, regional, and national cancer incidence, mortality, years of life lost, years lived with disability, and disability-adjusted life-years for 29 cancer groups, 1990 to 2016: A systematic analysis for the global burden of disease study. JAMA Oncol 4: 1553-1568, 2018.

3. Heist RS and Engelman JA: SnapShot: Non-small cell lung cancer. Cancer Cell 21: 448.e2, 2012.

4. Hirsch FR, Suda K, Wiens J and Bunn PA Jr: New and emerging targeted treatments in advanced non-small-cell lung cancer. Lancet 388: 1012-1024, 2016.

5. Fennell D, Summers Y, Cadranel J, Benepal T, Christoph D, Lal R, Das M, Maxwell F, Visseren-Grul C and Ferry D: Cisplatin in the modern era: The backbone of first-line chemotherapy for non-small cell lung cancer. Cancer Treat Rev 44: 42-50, 2016.

6. Arriagada R, Bergman B, Dunant A, Le Chevalier T, Pignon JP and Vansteenkiste J; International Adjuvant Lung Cancer Trial Collaborative Group: Cisplatin-based adjuvant chemotherapy in patients with completely resected non-small-cell lung cancer. $\mathrm{N}$ Engl J Med 350: 351-360, 2004.

7. Herbst RS, Morgensztern D and Boshoff C: The biology and management of non-small cell lung cancer. Nature 553: 446 , 2018.

8. Schmitz SU, Grote P and Herrmann BG: Mechanisms of long noncoding RNA function in development and disease. Cell Mol Life Sci 73: 2491-2509, 2016.

9. Ge P, Cao L, Yao YJ, Jing RJ, Wang W and Li HJ: lncRNA FOXD2-AS1 confers cisplatin resistance of non-small-cell lung cancer via regulation of miR185-5p-SIX1 axis. Onco Targets Ther 12: 6105-6117, 2019.

10. Liu MY, Li XQ, Gao TH, Cui Y, Ma N, Zhou Y and Zhang GJ Elevated HOTAIR expression associated with cisplatin resistance in non-small cell lung cancer patients. J Thorac Dis 8: 3314-3322, 2016.

11. Wang P, Chen D, Ma H and Li Y: LncRNA MEG3 enhances cisplatin sensitivity in non-small cell lung cancer by regulating miR-21-5p/SOX7 axis. Onco Targets Ther 10: 5137-5149, 2017.

12. Dragomir MP, Knutsen E and Calin GA: SnapShot: Unconventional miRNA functions. Cell 174: 1038-1038.e1, 2018.
13. Fujita Y, Yagishita S, Hagiwara K, Yoshioka Y, Kosaka N, Takeshita F, Fujiwara T, Tsuta K, Nokihara H, Tamura T, et al: The clinical relevance of the miR-197/CKS1B/STAT3-mediated PD-L1 network in chemoresistant non-small-cell lung cancer. Mol The 23: 717-727, 2015.

14. Bian B, Pan X, Yang JS, Wang ZX and De W: Upregulation of microRNA-451 increases cisplatin sensitivity of non-small cell lung cancer cell line (A549). J Exp Clin Cancer Res 30: 20, 2011.

15. Gao Y, Fan X, Li W, Ping W, Deng Y and Fu X: miR-138-5p reverses gefitinib resistance in non-small cell lung cancer cells via negatively regulating $G$ protein-coupled receptor 124 . Biochem Biophys Res Commun 446: 179-186, 2014.

16. Jin F, Wang Y, Li M, Zhu Y, Liang H, Wang C, Wang F, Zhang CY, Zen K and Li L: MiR-26 enhances chemosensitivity and promotes apoptosis of hepatocellular carcinoma cells through inhibiting autophagy. Cell Death Dis 8: e2540, 2017.

17. Liu Z, Shi A, Song D, Han B, Zhang Z, Ma L, Liu D and Fan Z: Resistin confers resistance to doxorubicin-induced apoptosis in human breast cancer cells through autophagy induction. Am J Cancer Res 7: 574-583, 2017.

18. Wang X, Lan Z, He J, Lai Q, Yao X, Li Q, Liu Y, Lai H, Gu C and Yan Q: LncRNA SNHG6 promotes chemoresistance through ULK1-induced autophagy by sponging miR-26a-5p in colorectal cancer cells. Cancer Cell Int 19: 234, 2019.

19. Zhao Z, Li J, Jiang Y, Xu W, Li X and Jing W: CLDN1 increases drug resistance of non-small cell lung cancer by activating autophagy via up-regulation of ULK1 phosphorylation. Med Sci Monit 23: 2906-2916, 2017.

20. Livak KJ and Schmittgen TD: Analysis of relative gene expression data using real-time quantitative PCR and the 2(-Delta Delta C(T)) method. Methods 25: 402-408, 2001.

21. Du Z, Sun T, Hacisuleyman E, Fei T, Wang X, Brown M, Rinn JL, Lee MG, Chen Y, Kantoff PW and Liu XS: Integrative analyses reveal a long noncoding RNA-mediated sponge regulatory network in prostate cancer. Nat Commun 7: 10982, 2016.

22. Kartha RV and Subramanian S: Competing endogenous RNAs (ceRNAs): New entrants to the intricacies of gene regulation. Front Genet 5: 8, 2014.

23. Deng H, Zhang J, Shi J, Guo Z, He C, Ding L, Tang JH and Hou Y: Role of long non-coding RNA in tumor drug resistance. Tumor Biol 37: 11623-11631, 2016.

24. Majidinia $M$ and Yousefi B: Long non-coding RNAs in cancer drug resistance development. DNA Repair (Amst) 45: 25-33, 2016.

25. Zheng ZG, Xu H, Suo SS, Xu XL, Ni MW, Gu LH, Chen W, Wang LY,Zhao Y and Tian B: The essential role of H19 contributing to cisplatin resistance by regulating glutathione metabolism in high-grade serous ovarian cancer. Sci Rep 6: 26093, 2016.

26. Han Z and Shi L: Long non-coding RNA LUCAT1 modulates methotrexate resistance in osteosarcoma via miR-200c/ABCB1 axis. Biochem Biophys Res Commun 495: 947-953, 2018.

27. Wang $\mathrm{W}$, Dong $\mathrm{M}$, Zhang $\mathrm{W}$ and Liu T: Long noncoding LUCAT1 promotes cisplatin resistance of non-small cell lung cancer by promoting IGF-2. Eur Rev Med Pharmacol Sci 23: 5229-5234, 2019.

28. Galluzzi L, Pietrocola F, Bravo-San Pedro JM, Amaravadi RK, Baehrecke EH, Cecconi F, Codogno P, Debnath J, Gewirtz DA, Karantza V, et al: Autophagy in malignant transformation and cancer progression. EMBO J 34: 856-880, 2015.

29. Yang Y, Jiang C, Yang Y, Guo L, Huang J, Liu X, Wu C and Zou J: Silencing of LncRNA-HOTAIR decreases drug resistance of Non-small cell lung cancer cells by inactivating autophagy via suppressing the phosphorylation of ULK1. Biochem Biophys Res Commun 497: 1003-1010, 2018.

30. Xiong H, Ni Z, He J, Jiang S, Li X, Gong W, Zheng L, Chen S, Li B and Zhang N: LncRNA HULC triggers autophagy via stabilizing Sirtl and attenuates the chemosensitivity of HCC cells. Oncogene 36: 3528, 2017.

31. Jin L, Li Y, Zhang Z, He T, Hu J, Liu J, Chen M, Gui Y, Yang S and Mao X: miR-514a-3p functions as a tumor suppressor in renal cell carcinoma. Oncol Lett 14: 5624-5630, 2017.

32. Kim J, Kundu M, Viollet B and Guan KL: AMPK and mTOR regulate autophagy through direct phosphorylation of Ulk1. Nat Cell Biol 13: 132, 2011.

33. Tang F, Hu P, Yang Z, Xue C, Gong J, Sun S, Shi L, Zhang S, Li Z and Yang C: SBI0206965, a novel inhibitor of Ulk1, suppresses non-small cell lung cancer cell growth by modulating both autophagy and apoptosis pathways. Oncol Rep 37: 3449-3458, 2017. 